\title{
A Transmission Power Self-Optimization Technique for Wireless Sensor Networks
}

\author{
F. Lavratti, ${ }^{1}$ A. Ceratti, ${ }^{1}$ D. Prestes, ${ }^{1}$ A. R. Pinto, ${ }^{2}$ L. Bolzani, ${ }^{1}$ F. Vargas, ${ }^{1}$ C. Montez, ${ }^{3}$ \\ F. Hernandez, ${ }^{4}$ E. Gatti, ${ }^{5}$ and C. Silva ${ }^{6}$ \\ ${ }^{1}$ Signals \& Systems for Computing Group (SiSC), Catholic University of Rio Grande do Sul (PUCRS), 90619-900 Porto Alegre, \\ RS, Brazil \\ ${ }^{2}$ DCCE, Universidade Estadual Paulista (UNESP), 15054-000 São José do Rio Preto, SP, Brazil \\ ${ }^{3}$ PGEAS, Universidade Federal de Santa Catarina (UFSC), 88040-900 Florianópolis, SC, Brazil \\ ${ }^{4}$ Unidad Reguladora de Servicios de Comunicaciones (URSEC), Universidad ORT, 11300 Montevideo, Uruguay \\ ${ }^{5}$ Instituto Nacional de Tecnologia Industrial (INTI), C1001AAF Buenos Aires, Argentina \\ ${ }^{6}$ Pontificia Universidad Católica del Perú (PUCP), Lima 32, Peru \\ Correspondence should be addressed to L. Bolzani, leticia@poehls.com
}

Received 7 November 2011; Accepted 19 December 2011

Academic Editor: F. Vasques

Copyright () 2012 F. Lavratti et al. This is an open access article distributed under the Creative Commons Attribution License, which permits unrestricted use, distribution, and reproduction in any medium, provided the original work is properly cited.

Wireless sensor networks (WSNs) are generally used to monitor hazardous events in inaccessible areas. Thus, on one hand, it is preferable to assure the adoption of the minimum transmission power in order to extend as much as possible the WSNs lifetime. On the other hand, it is crucial to guarantee that the transmitted data is correctly received by the other nodes. Thus, trading off power optimization and reliability insurance has become one of the most important concerns when dealing with modern systems based on WSN. In this context, we present a transmission power self-optimization (TPSO) technique for WSNs. The TPSO technique consists of an algorithm able to guarantee the connectivity as well as an equally high quality of service (QoS), concentrating on the WSNs efficiency $(E f)$, while optimizing the transmission power necessary for data communication. Thus, the main idea behind the proposed approach is to trade off WSNs $E f$ against energy consumption in an environment with inherent noise. Experimental results with different types of noise and electromagnetic interference (EMI) have been explored in order to demonstrate the effectiveness of the TPSO technique.

\section{Introduction}

Recent advancements in wireless communication and electronic technology have made possible the development of small, low-cost, low-power, and multifunctional sensor nodes $[1,2]$. Wireless sensor networks (WSNs) are composed of communication nodes, which contain sensing, data processing, and communication components as well as power supply, typically a battery. In more detail, these nodes are able to collect different types of data and to communicate with each other. Nowadays, WSNs have been increasingly deployed for both civil and military applications which typically work in harsh environments. Considering sensor nodes, resources like processor, memory, and battery are generally restricted, since their replacement is considered prohibitive due to the hazardous and inaccessible places where they are supposed to operate [3]. In this scenario, where nodes are likely to operate on limited battery life, power conservation can be considered one of the most important issues [4]. Transmitting at unnecessary high power not only reduces the lifetime of the WSNs nodes but also introduces excessive interference. Thus, transmitting at the lowest possible power while preserving the network connectivity and reliability has become crucial points related to WSNs $[4,5]$. In this paper, $E f$ is defined as the number of received messages by the master node (MN) in relation to the estimated number of sent messages by the slave nodes (SNs).

It is important to point out that communication reliability in WSNs can be degraded by different interference sources, such as WLAN, Bluetooth, or any other device that 
shares the $2.4 \mathrm{GHz}$ bandwidth. In addition to this bandwidth, the always increasing population of AM/FM radio devices is also responsible for increasing the environment noise hostility [6]. This evokes some concerns about the robustness of sensor network communications and can limit the wide adoption of WSN technology by the industry.

In this paper, we present a transmission power selfoptimization (TPSO) technique able to adjust individually the transmission power of each sensor node that composes the WSN, in order to guarantee a predefined efficiency $(E f)$ for the network $[7,8]$. The TPSO technique aims at guaranteeing the lowest possible transmission power while maintaining the connectivity of the WSN as well as the reliability of the transmitted data. Thus, the main idea behind the selfoptimization algorithm is to assure the trade-off between WSNs Ef and data transmission energy consumption. The proposed approach trades off the longest WSNs lifetime against the highest possible efficiency, and vice versa. To do so, this work presents a complete evaluation of the TPSO technique's effectiveness in different scenarios that exploit three types of noise considered inherent to the environment: (1) it exploits the distance variation between network nodes, (2) it exploits conducted EMI noise affecting the WSN, and (3) it exploits radiated EMI noise affecting the WSN. The effectiveness of the proposed technique has been evaluated using a suitable developed case study composed of eight SNs that communicate with one MN in a star-topology network. The main goals of this paper are.

(1) To provide experimental results showing the impact of the previously mentioned sources of noise on the WSNs communication. In more detail, to demonstrate that the distance variation between the nodes composing the WSN as well as the conducted and irradiated EMI do degrade the WSNs Ef. Consequently, the presence of any of these types of noise or any combination of them increases the necessity to communicate using a higher transmission power level in order to maintain the connectivity of the WSN as well as the WSNs Ef under expected values.

(2) To demonstrate the effectiveness of the TPSO technique, by showing that it can guarantee the targeted WSNs Ef in a time-variant noise environment, while decreasing the energy necessary for data transmission between nodes.

The obtained results during the experiments quantified the impact of the previously mentioned interference sources in WSNs and demonstrate the effectiveness of the proposed solution. In this sense, the experimental results show that the TPSO technique significantly reduces the energy consumption with respect to the data transmission, while maintaining the targeted WSNs Ef.

This paper has been organized as follows. Section 2 summarizes the background related to WSN concerns. Section 3 presents the TPSO technique, detailing the communication model as well as the self-optimization algorithm. Section 4 presents the case study adopted for experiments. This section also details the three different scenarios used for noise injection on the WSN and summarizes the obtained results. Finally, Section 5 draws the conclusions.

\section{Background}

Nowadays, the adoption of WSNs in several civil and military scenarios has become a highly viable solution when aiming at the transmission of collected data in inaccessible places. However, the sensor nodes are usually powered by battery and consequently its preservation in order to increase the WSNs lifetime is considered crucial. Thus, the fact that WSNs are applied in locations where the access is difficult and replacing the battery would often not be feasible defines the goal of long battery life as essential to such networks [3]. Moreover, the reliability of the data collected, processed, and delivered has to be assured. As a result, high reliability and low energy consumption have become important issues when dealing with WSNs. In this context, several techniques have been proposed in order to deal with the previously mentioned concerns.

Concerning reliability, WSNs are required to collect, process, and deliver information interacting with the environment where they are placed. Thus, due to uncontrolled environment inherent noise, it does not surprise that WSNs frequently show low connectivity levels demonstrating poor quality of service (QoS) [9]. One strategy to cope with the QoS requirements is to adopt data fusion techniques. In dense networks, they are used in order to increase the sensor's reading dependability to achieve a more accurate estimation of the environment and finally to assure a longer network lifetime [3]. In these approaches, sensed scalars are sent to master nodes (MNs) which themselves fuse the data with the objective to extract useful information from a set of readings.

Another factor that interferes with the QoS is the increasing number of nodes that compose WSNs. This fact leads to a high complexity of the systems and the impossibility of human administration. Facing these problems, the development of computing systems that do not need human intervention to operate correctly has emerged. Thus, systems with so-called self-management characteristics, computer systems that are able to manage themselves based on high-level objectives given by the administrators before the beginning of the WSNs operation have been developed to cope with the increasing complexity [10]. The approach described in this work is considered to regulate the transmission power level and, therefore, manage a part of the administration itself.

Due to the fact that all sensor nodes composing the network are battery powered, the above, mentioned reliability concerns are desired to be developed with strategies that also aim at the minimization of the transmission power. In other words, power conservation strategies can help to prolong the lifetime of the sensor nodes and consequently of the WSN as a whole. Higher transmission power enhances the connectivity among the entire network and improves its capacity, but leads to faster exhaustion of the batteries. Indeed, transmitting at unnecessary high power not only reduces the lifetime of the WSN but also introduces excessive interference. It is in the network designer's best interest to 
have each node transmit at the lowest possible power while preserving network connectivity. It is a desirable characteristic, but it is difficult to modify the transmission power after the system's deployment. However, transmitting at low transmission power can compromise the connectivity and consequently the reliability of the transmitted data. As a result, the development of such systems has to deal with a trade-off between the WSNs lifetime and its reliability. In other words, a high transmission power would guarantee a high reliable system but lead to shorter system lifetime. A low transmission power is able to make the component's battery last for a longer period, but the system may not work properly or without a satisfying reliability.

Finally, the reliability and the energy consumption represent decisive factors for the choice of the transmission power of the WSN. Indeed, different factors can affect the definition of the optimal transmission power. The number and the distances between the sensor nodes deployed can interfere drastically in the obtained reliability of the network. In more detail, an increased density of the network deployed requires a lower transmission power.

Thus, as a result of the previously mentioned concerns, choosing the optimal transmission power has become an important issue for WSNs. In [4], a suitable criterion for a optimal transmission power has been introduced. One of the goals of forming a network is to have connectivity, in other words, each node should be able to communicate with the other ones. The connectivity level of the ad hoc wireless network depends on the transmission power of the nodes. If the transmission power is too small, the network might be disconnected. However, transmitting at excessively high power is inefficient due to mutual interferences in the shared radio channel and the decreased battery lifetime. Thus, it is intuitively clear that the optimal transmission power is the minimum power sufficient to guarantee the network's connectivity. Ideally, the transmission power of a node should be adjusted on a link-by-link basis to achieve the maximum possible power savings [4]. Nonetheless, due to the absence of a central controller in a pure ad hoc network with flat architecture, performing power control on a linkby-link basis is a complicated task. A more feasible solution for the implementation of the WSN is to have all the nodes use the same transmission power.

To conclude, it is important to highlight that none of the above-mentioned approaches is able to adjust the transmission power level of the operating WSN. By choosing the transmission power level before inserting the nodes into an unpredictable environment, these techniques are not able to guarantee the trade-off between QoS and battery life.

\section{The Proposed Technique}

The transmission power self-optimization (TPSO) technique basically deals with the trade-off between WSNs Ef and energy consumption. In what follows, we will describe the communication model adopted and the self-optimization algorithm.
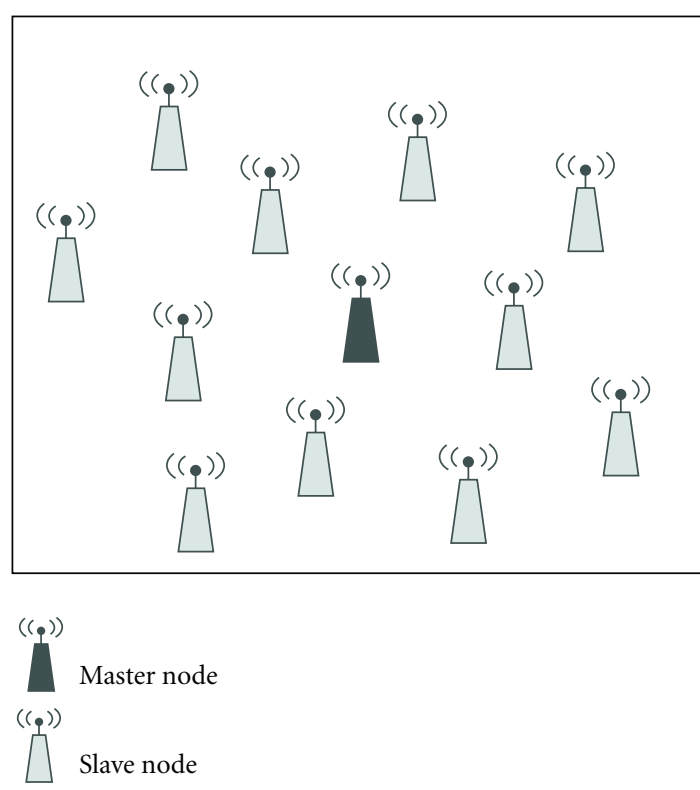

Figure 1: WSN model.

3.1. Communication Model. The adopted model considers one master node (MN), also called base station, and $n$ Slave nodes (SNs) according to Figure 1. In more detail, the data collected by the SNs is sent to the MN that performs data fusion. All the $\mathrm{SNs}$ reach the master using just one hop.

In this work, the concept of session monitoring is adopted. A session $S$ is composed of $t$ session time (ST) rounds with the length $R$. Therefore, $S$ is composed of $\mathrm{ST}_{0}, \mathrm{ST}_{1}, \mathrm{ST}_{2}, \ldots, \mathrm{ST}_{(t-1)}$. The round concept is used to synchronize nodes, and it also represents the periodicity of the data fusion task [3].

Regarding the MN, it performs the data fusion considering only the messages that arrived on time. In this particular work, the MN only fuses data that arrived within the same session. Thus, the number of required messages, the round time, (RT) and the session time (ST) parameters are sent by the $\mathrm{MN}$ at the beginning of each session, forming the so-called checkpoint [3]. Moreover, the MN computes the performance metrics during the checkpoint round in order to adjust the WSN.

In the communication model adopted in this work, we considered the efficiency $(E f)$ metric. In more detail, $E f$ is measured considering the number of received messages by the MN before finishing the session in execution in relation to the estimated number of sent messages by the SNs. Thus, Ef is calculated according to the following equation:

$$
E f=\frac{\sum_{i=1}^{N} M r_{i}}{E_{M s}}
$$

where $N$ is the number of rounds, $M r$ is the number of received messages, and $E_{M s}$ is the estimative of the sent messages by the SNs. In more detail, $E_{M s}$ is given by (2) where $K$ represents the number of required messages by the $\mathrm{MN}$ and $D e$ is the SN's density in the considered WSN. Thus, this 


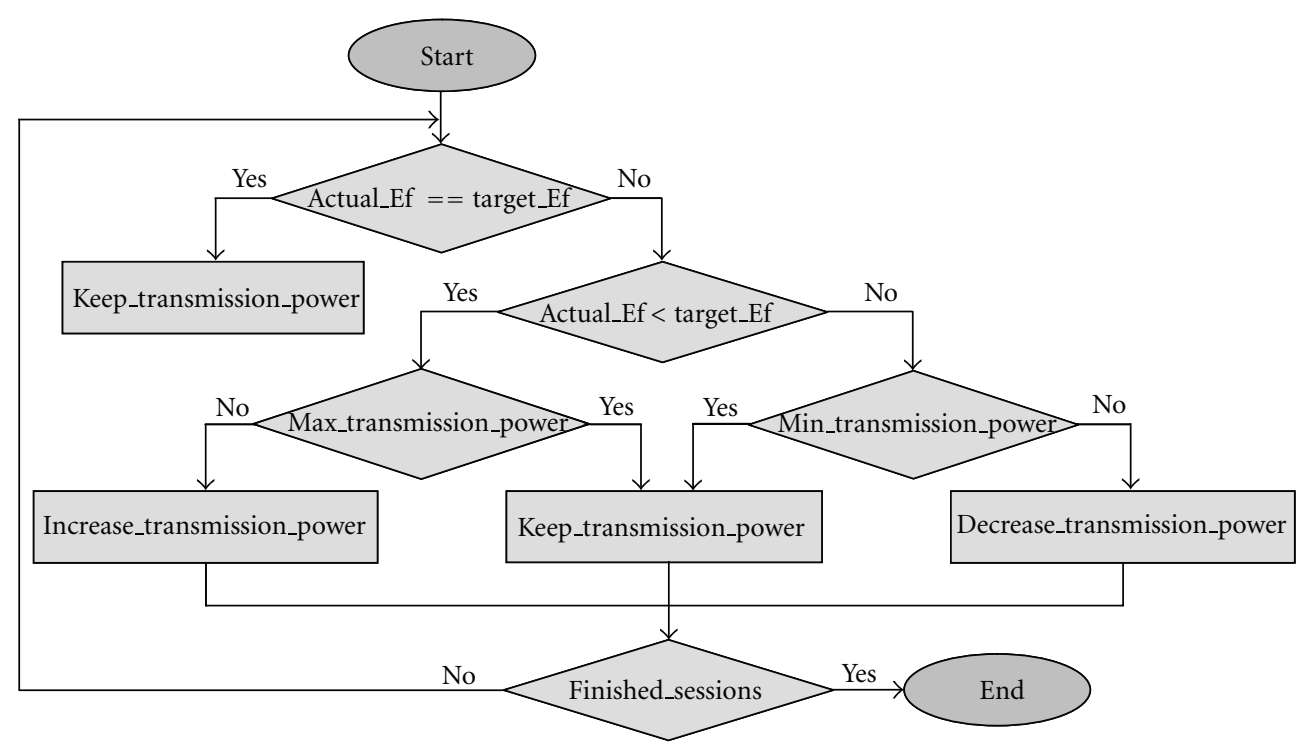

FIGURE 2: TPSO algorithm.

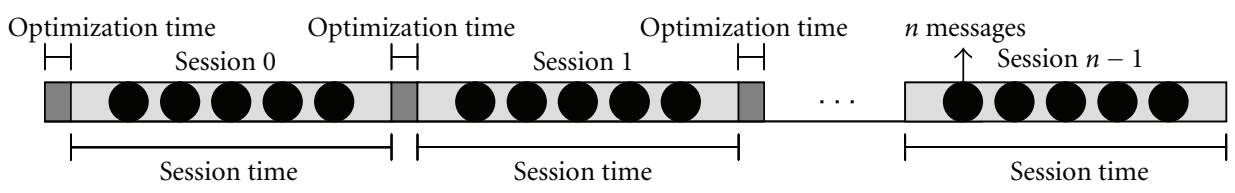

Figure 3: Conceptual idea of the proposed technique.

metric indicates how many messages are used during data fusion task:

$$
E_{M s}=K \times D e \times N .
$$

3.2. The Transmission Power Self-Optimization Technique. The TPSO technique is based on a simple and decentralized algorithm that runs on the application layer of the WSN $[7,8]$. The main idea behind the TPSO technique is to adjust the transmission power taking into consideration the entire WSNs Ef. To do so, the TPSO technique uses the Ef associated to each $\mathrm{SN}$ to compute the WSNs Ef. In more detail, the $\mathrm{MN}$ is responsible to compute the WSNs Ef as well as the $E f$ associated to each $\mathrm{SN}$ and to send the specific $E f$ to each SN. Finally, the SNs are in charge of adjusting their own transmission power levels based on their Ef by performing the TPSO algorithm. Figure 2 depicts the block diagram of the self-optimization algorithm.

Observing the block diagram shown in Figure 2, it is possible to note that the transmission power is adjusted by comparing the actual_Ef, computed at the end of each session, and the target_Ef, set at the beginning of the communication. In more detail, the actual_Ef is computed using the data collected during the ST, that is, while the set of messages that composes the considered session are sent. Regarding the target_Ef, it is important to note that the value set at the beginning of the communication has a tolerance of $5 \%$, which means setting a target_Ef of $90 \%$, this value oscillates from $85 \%$ to $95 \%$. Moreover, it is important to highlight that the sensor nodes have a predefined minimum and maximum transmission power, which cannot be exchanged or overwritten by the algorithm. Thus, the TPSO technique increases or decreases the transmission power step-by-step passing through all the transmission power levels available for each node. Finally, the self-optimization algorithm is performed by the SNs that compose the WSN and it can be adopted in WSNs that do not provide any type of transmission power optimization.

Figure 3 shows the conceptual idea of the TPSO technique related to the temporal execution of the self-optimization algorithm. Observing Figure 3, it is possible to see between two consecutive sessions that the adjustment of the transmission power is performed during the so-called optimization time. In detail, the MN computes the Ef associated to each SN during the optimization time and sends these values to them. The algorithm running on the $\mathrm{SN}$ then adjusts the transmission power level according to the actual_Ef when it is necessary.

\section{Experimental Results}

This section evaluates the effectiveness of the TPSO technique using different types of interference mechanisms. The experiments have been performed considering three different scenarios divided as follows: Scenario 1 evaluates the impact of the distance variation between the $\mathrm{MN}$ and SNs on the communication efficiency. Scenario 2 exploits the use 


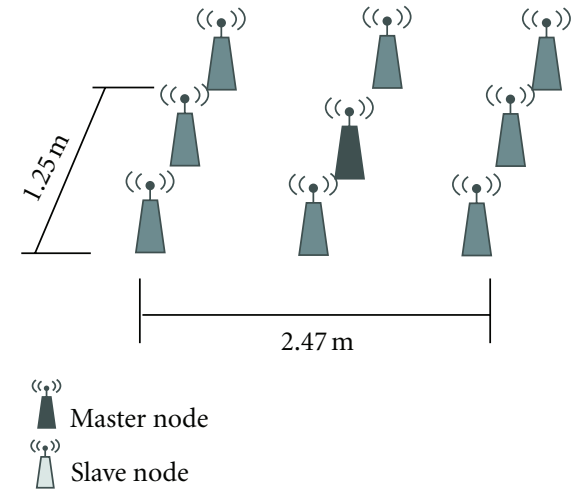

Figure 4: Case study adopted.

of conducted EMI to generate power supply disturbances (PSDs) able to degrade the WSNs communication. Finally, Scenario 3 uses irradiated EMI based on AM/FM or WiFi noise to affect the WSN. Thus, the main goals of these experiments have been twofold: (1) to demonstrate the impact of the different types of interference on the WSNs Ef and (2) to demonstrate the effectiveness of the TPSO technique in order to maintain the target_Ef when different types of interferences affect the WSN.

4.1. Case Study. In order to demonstrate the TPSO technique's effectiveness with respect to the three scenarios mentioned above, we developed a case study composed of nine sensor nodes as shown in Figure 4.

Observing Figure 4, it is possible to see that the case study adopted is composed of one master node (MN) and eight slave nodes (SNs) performing the following tasks.

(i) Master node (MN): it starts the communication with the SNs and performs the fusion of the received data. It is also in charge of computing the $E f$ of each $\mathrm{SN}$ and sending them the parameters necessary to perform the transmission power optimization.

(ii) Slave node $(S N)$ : it sends data messages to the $\mathrm{MN}$ and executes the TPSO algorithm in order to optimize the transmission power based on the parameters sent by the MN.

It is important to point out that both $\mathrm{MN}$ and $\mathrm{SNs}$ are XBee PRO modules, which use the IEEE 802.15.4 networking protocol for fast point-to-multipoint or peer-to-peer networking [11]. The IEEE 802.15.4 is a standard that specifies the lower two layers of the wireless communication protocol: (1) the physical layer (PHY) and (2) the media access control (MAC). Indeed, the IEEE 802.15.4 supports the unique needs of low-cost, low-power, and low-rate WSN. The PHY layer can operate with $250 \mathrm{Kbps}$ of maximum transmission rate. Regarding the MAC, it supports two types of operating modes: (1) beaconless mode, a nonslotted carrier sense multiple access collision avoidance (CSMA/CA) and (2) beacon mode, where beacons are sent periodically by a PAN coordinator [12]. In the latter case, nodes are synchronized by a superframe structure. Finally, the XBee PRO modules can be
TABLE 1: Transmission power levels.

\begin{tabular}{lc}
\hline Level & XBee PRO $[\mathrm{dBm}]$ \\
\hline 0 & 10 \\
1 & 12 \\
2 & 14 \\
3 & 16 \\
4 & 18 \\
\hline
\end{tabular}

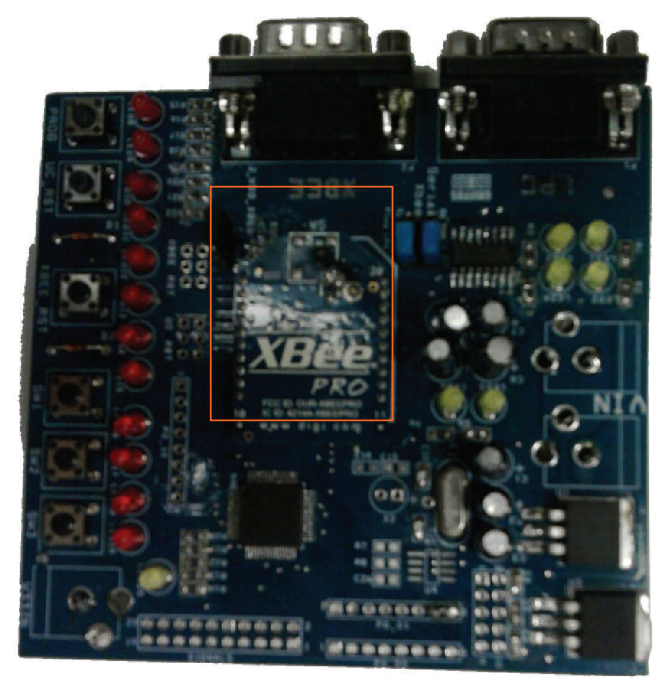

Figure 5: PCB of the WSNs nodes.

configured to operate using five different transmission power levels that range from 0 to 4 . Table 1 summarizes the transmission power levels of the XBee PRO module and their respective communication power in $\mathrm{dBm}$. Observing this table, it is possible to see that the minimum transmission power level corresponds to $10 \mathrm{dBm}$ and the maximum to $18 \mathrm{dBm}$.

Figure 5 shows the printed circuit board (PCB) of a wireless sensor ( $\mathrm{MN}$ or $\mathrm{SN})$. This board was designed by the Computing Signals and Systems Group (SiSC) of the Catholic University (PUCRS) and was used during all the experiments. It is basically built around two major components: the XBee PRO module and the ARM 7 microprocessor, which runs the self-optimization algorithm.

4.2. Scenario 1. Scenario 1 aims to analyze the impact of distance variation between SNs and the MN on the WSNs Ef as well as to demonstrate the effectiveness of the proposed approach in order to maintain a target_Ef by automatically increasing the transmission power of SNs when necessary. Thus, first of all, the case study described previously has been analyzed in an environment exposed to real noise where one of the SNs has been moved from an initial position with respect to the $\mathrm{MN}$ to a different final position further away from the MN. In more detail, the distance between one of the SNs and the MN has been increased step-by-step and the behavior of the WSN has been analyzed in terms of Ef and energy consumption of the WSN. In this scenario, an "experiment" was defined as a set of 72 sessions, each 


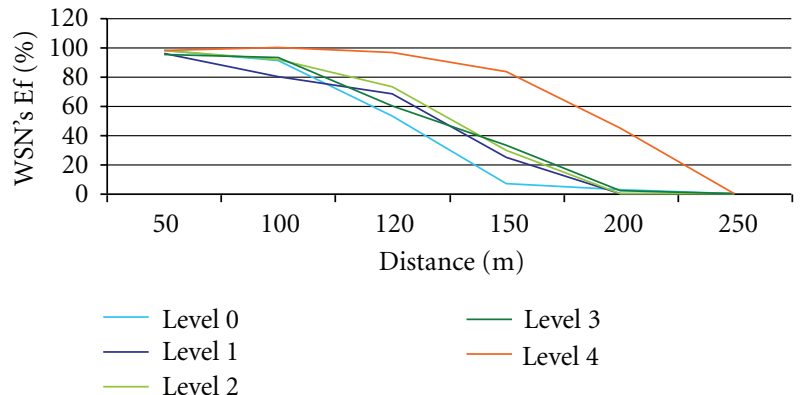

Figure 6: Scenario 1. Distance impact on WSNs Ef and energy consumption considering the five different transmission power levels of the XBee PRO module.

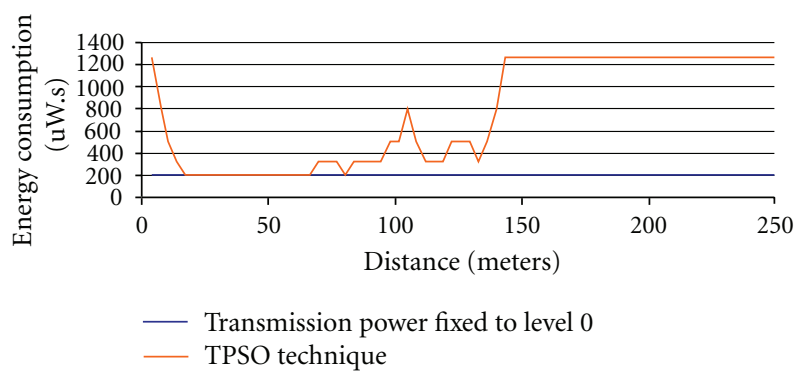

Figure 7: Scenario 1. Comparison of energy consumption obtained using transmission power level fixed to 0 and the TPSO algorithm.

one composed of 20 messages. The session time has been configured to be of $10 \mathrm{~s}$ and the optimization time to be $0.5 \mathrm{~s}$.

Figure 6 depicts results for one SN moving far away from the MN considering six steps of the distance variation. In this sense, Figure 6 analyzes the WSNs $E f$ considering distances of $50,100,120,150,200$, and 250 meters with respect to the original position of the $\mathrm{SN}$. For each previously mentioned distance, we considered the five different transmission power levels for the XBee PRO module ranging from 0 to 4 .

Observing Figure 6, we can see that the WSNs Ef decreases when the distance between the MN and the SN increases demonstrating the negative impact of the increasing distance on the WSNs Ef.

For the following graphs, we considered Scenario 1 as described earlier. In more detail, the distances assumed values from 0 to 250 meters, the target Ef was set to $90 \%$ and the transmission power level was fixed to 0 or to 4 . It is important to highlight that setting target Ef to $90 \%$ means that a tolerance of $5 \%$ is allowed. Figures 7 and 8 depict the results obtained by setting the transmission power level to 0 . Figure 7 compares the energy consumed as a function of distance comparing the fixed 0-level transmission power with the one associated to the TPSO technique. Figure 8 compares the WSNs Ef while using a fixed 0-level transmission power and while adopting the TPSO algorithm.

The average $E f$ of the WSN using the 0-level fixed transmission power was measured to be $49.59 \%$, while using the TPSO algorithm such value increased to $62.81 \%$. This corresponds to an increase of $13.21 \%$. At the same time,

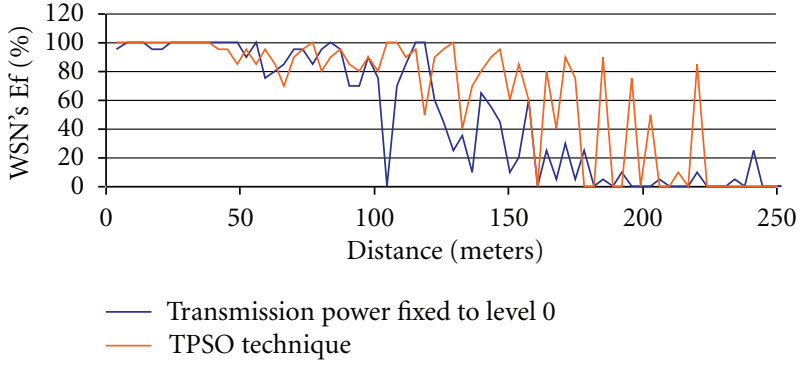

Figure 8: Scenario 1. Comparison between the WSNs Ef obtained using transmission power level fixed to 0 and the one associated to the TPSO algorithm.

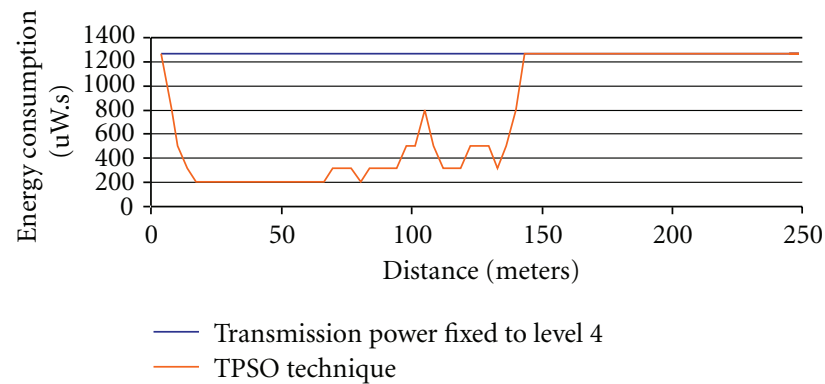

FIGURE 9: Scenario 1. Comparison of energy consumption obtained using transmission power level fixed to 4 and the TPSO algorithm.

the power consumption of the proposed technique is about $285 \%$ higher than the power consumed by the WSN to transmit at fixed 0-level. Therefore, observing Figures 7 and 8, one can conclude that the TPSO achieves a higher Ef consuming more power. For applications where efficiency is a key-requirement and must be guaranteed even consuming more energy, the proposed TPSO technique is a good alternative.

It should also be observed that neither of the two configurations achieved the aimed efficiency level of $90 \%$. This is attributed to the fact that when the distance between the MN and the SN increased beyond 150 meters, there was almost no connectivity between them. Therefore, even transmitting at the highest power level (4), the TPSO is not able to compensate the impact of the distance (even though the communication efficiency is higher with TPSO technique than without it). This is a typical case of transmission power saturation. Thus, the high energy consumption increase of the WSN with the TPSO technique can be attributed to the fact that the maximum transmission power is maintained until the end of the experiment, even if the WSNs $E f$ could not be further improved due to the high distance between $\mathrm{MN}$ and $\mathrm{SN}$.

Figures 9 and 10 are similar to Figures 7 and 8, except for the fact that instead of using the lowest transmission level (0), Figures 9 and 10 depict results for the highest transmission level (4). Figure 9 compares the energy consumption associated to WSN using the proposed technique and WSN using the transmission power fixed to level (4). Figure 10 


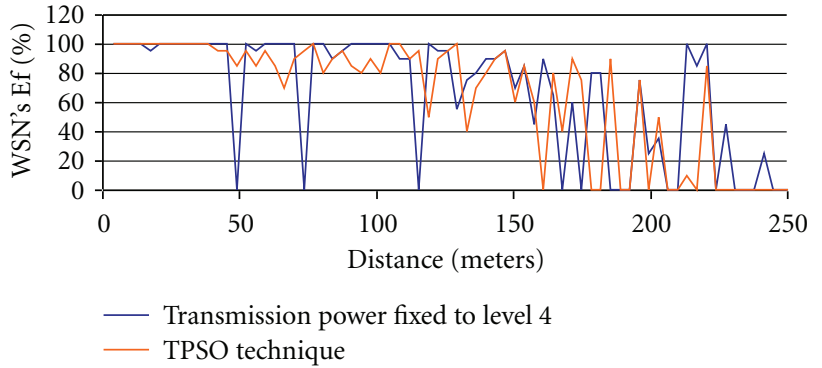

Figure 10: Scenario 1. Comparison of WSNs Ef obtained using transmission power level fixed to 4 and the TPSO algorithm.

demonstrates the WSNs Ef while adopting the proposed algorithm and while using the fixed transmission power level.

The results depicted in Figures 9 and 10 clearly demonstrate the efficiency of the TPSO technique. When the distance between the $\mathrm{SN}$ and $\mathrm{MN}$ increases, it is possible to observe that the energy consumption increases in order to maintain the target WSNs $E f$. It is worth noting that the energy consumption is associated with the transmission power level adopted during the communication between the $\mathrm{MN}$ and SNs. In more detail, the WSNs Ef with the fixed transmission power level 4 is $65.68 \%$ whereas the TPSO yields $62.81 \%$, or of $2.87 \%$ less than the one using the maximum transmission power level. In addition, it should be noted that the energy consumption of the proposed approach is $38.87 \%$ lower when compared to the fixed transmission power level 4 . Thus, one can conclude that the TPSO technique saves more than a third in energy while loosing less than $3 \%$ of efficiency to communicate.

4.3. Scenario 2. Scenario 2 exploits the use of power supply disturbances (PSD) in order to generate conducted EMI on the WSN. During this phase, we used suitable developed equipment able to generate PSD according to the IEC 610004-29 standard [13]. This IEC standard defines rules for immunity testing to voltage dips, short interruptions, and voltage variations on d.c. input power port of integrated circuits and systems. Due to the large possible range, the voltage dips' percentage that can be assumed during the experiments, an initial experiment has been performed in order to find the maximum variation supported by the WSN nodes. The results obtained showed that the WSN nodes are able to maintain the connectivity between $\mathrm{MN}$ and $\mathrm{SN}$ up to the limit of $16 \%$ of voltage dip with respect to nominal $V d d$ of 3.3 V. During the experiments, the PSD's frequency has been set to $500 \mathrm{~Hz}$.

Finally, it is important to point out that an experiment is defined as a set of 50 sessions, each one composed of the same 10 messages. These 10 messages are sent during each session by the SNs to the MN. Indeed, the session time has been configured to be of $10 \mathrm{~s}$ and the optimization time to be of $0.5 \mathrm{~s}$. Figure 11 depicts the equipment used to generate noise in the form of voltage dips at the $V d d$ input pins of the nodes composing the WSN. This waveform was captured with an oscilloscope. It is important to underline that no noise was

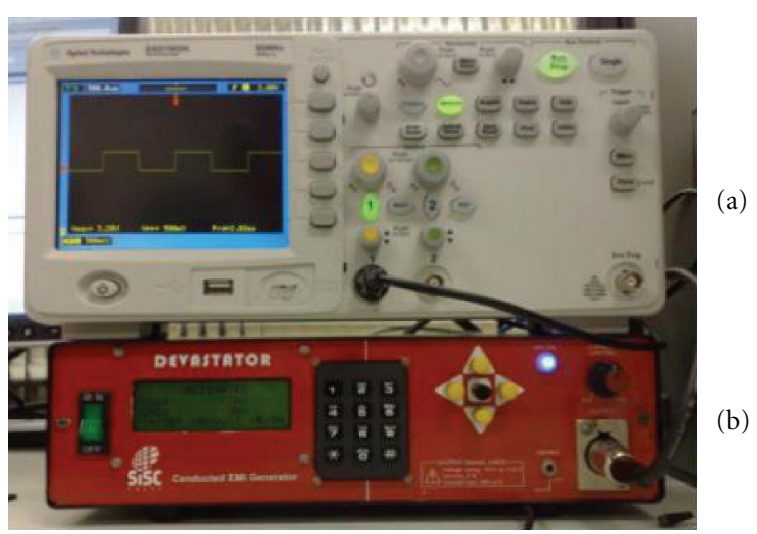

FIgURE 11: Scenario 2. (a) IEC 61000-4-29 compliant noise injected at the $V d d$ input port of the WSN nodes; (b) Noise generator developed by the SiSC-PUCRS Group [14].

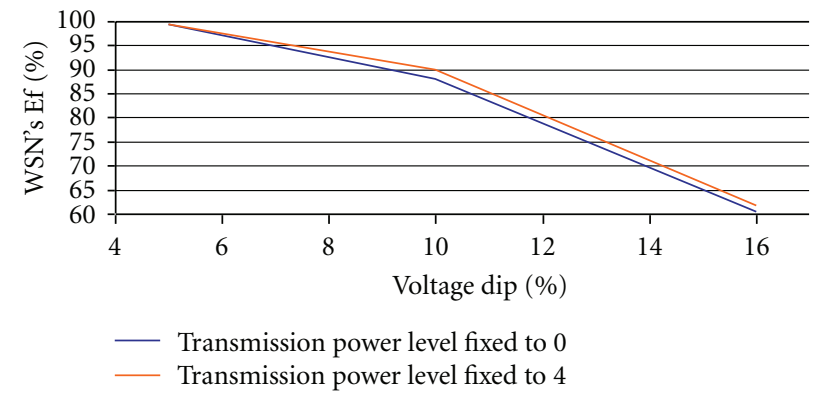

FIgURE 12: Scenario 2. Conducted EMI impact on WSNs Ef and energy consumption considering the five different transmission power levels of the Xbee PRO module.

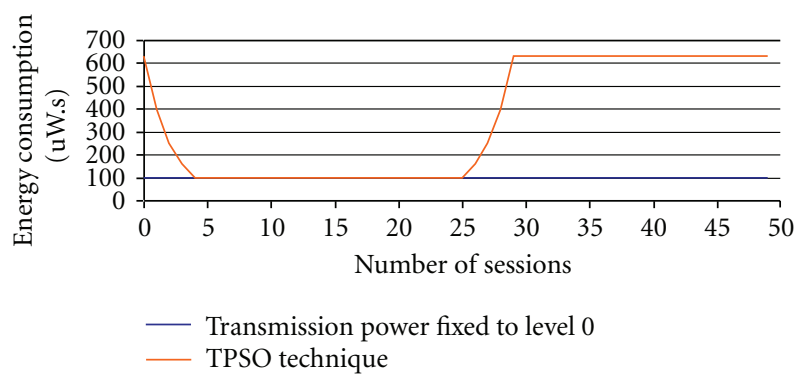

FIGURE 13: Scenario 2. Comparison of energy consumption considering the transmission power fixed at level 0 and the WSN with the TPSO technique.

injected during the first 25 sessions, whereas voltage dips have been injected during the execution of the sessions 25 to 49 .

Figure 12 shows the impact of the PSD on the WSNs $E f$. Observing this figure, it is possible to conclude that increasing the percentage of voltage dip decreases significantly the WSNs Ef.

The results obtained with respect to PSD in Scenario 2 have been summarized in Figures 13-16. Figure 13 compares the energy consumption and Figure 14 compares the Ef of 


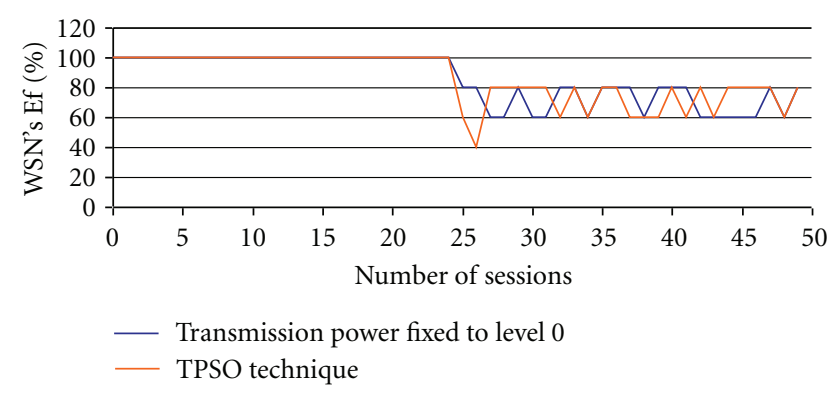

Figure 14: Scenario 2. Comparison of WSNs Ef considering the transmission power fixed at level 0 and the WSN with the TPSO technique.

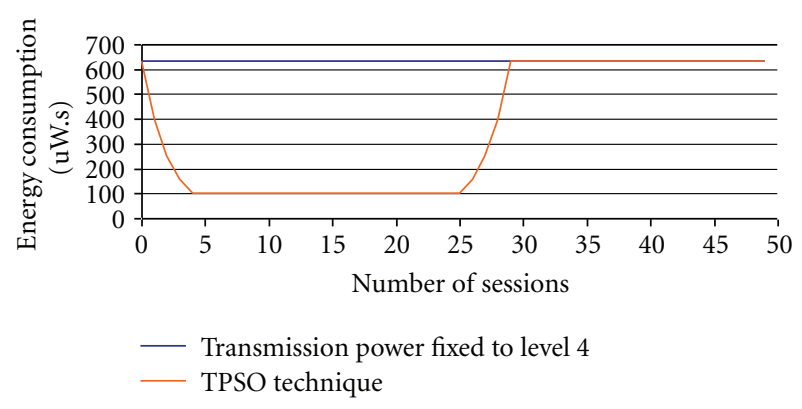

Figure 15: Scenario 2. Comparison of energy consumption considering the transmission power fixed at level 4 and the WSN with the TPSO technique.

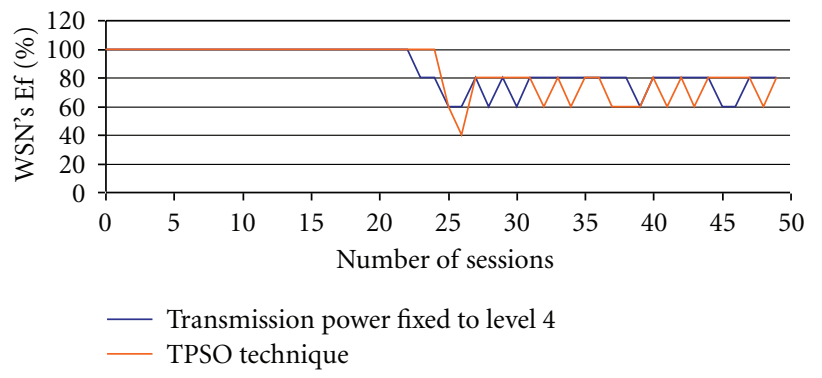

Figure 16: Scenario 2. Comparison of WSNs Ef considering the transmission power fixed at level 4 and the WSN with the TPSO technique.

WSNs with and without the TPSO technique. The WSN without the proposed self-optimization algorithm has been set to ensure communication between nodes using the fixed minimum transmission power level, level 0 in Table 1, and the WSN with TPSO assumed the target Ef $=90 \%$. Observing these figures, it is possible to conclude that the average WSNs Ef guaranteed by the WSN with and without the TPSO technique was of $85 \%$ and $84 \%$, respectively. Note that the energy consumed by the WSN with the TPSO technique was higher than the one consumed by the WSN communicating at the minimum transmission power level. This can be justified by the fact that the WSN with the proposed approach increases the transmission power level in order to guarantee the target_Ef of $90 \%$. It can also be observed in Figure 14 that with voltage dips set to maximum allowed value of $16 \%$, the noise is so strong that a saturation
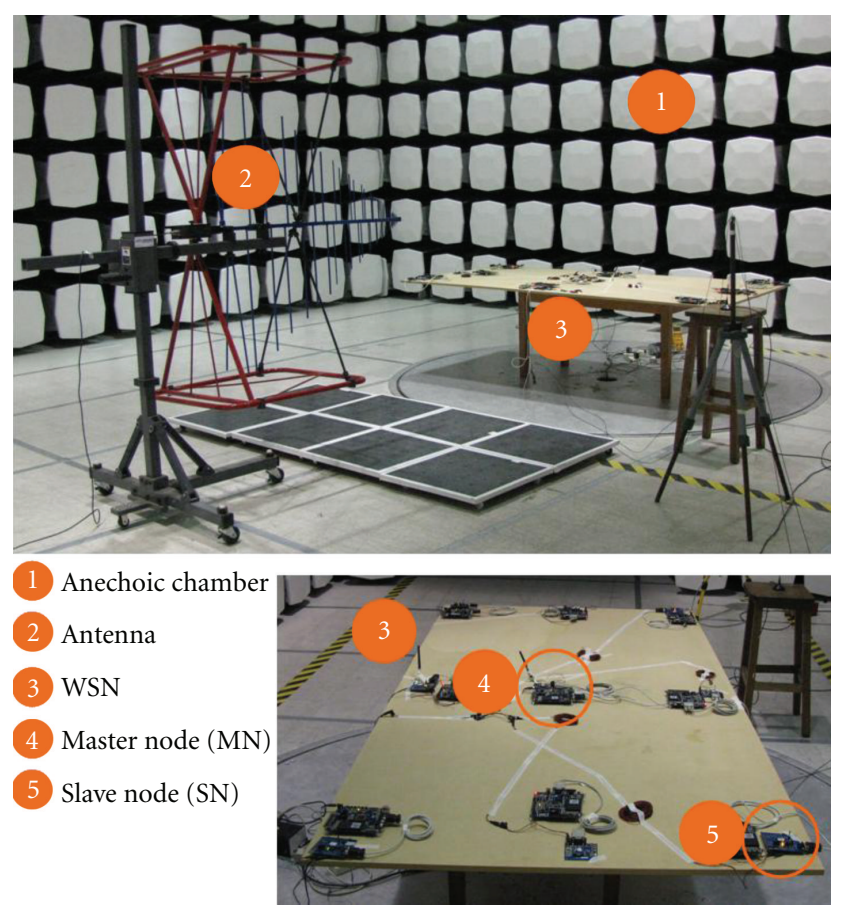

FIgURE 17: EMI environment adopted.

of the transmission power can be observed from session 28 onwards. Here even the transmission power level set to its maximum is not able to raise the WSNs Ef above a level of about $70 \%$.

Now looking at Figure 15, the graph is showing the comparison of the energy consumption of a WSN with the transmission power level fixed to the maximum value and an WSN with the proposed technique applied. The graph of Figure 16 shows the comparison of the same two WSN in respect to their Ef. In media, the Ef of the maximum transmission level WSN is of $86 \%$ and the WSN using the proposed approach is reaching $85.5 \%$. Thus, we can conclude that the TPSO technique is practically maintaining the same reliability, while it is saving more than $55 \%$ of energy consumption.

4.4. Scenario 3. The main goal of the experiments performed in Scenario 3 was to demonstrate the impact of irradiated EMI on the WSNs Ef. The experiments have been performed using an anechoic chamber and an antenna which irradiates noise over the WSN. Figure 17 depicts the EMI environment adopted where it is possible to see the antenna and the WSN placed on the table inside the anechoic chamber according to the case study's diagram presented in Figure 4. In more detail, the WSN has been exposed to two different types of irradiated EMI. The first type concerns AM/FM noise that has been irradiated over the WSN according to the following characteristics:

(i) carrier frequency of $2.4093 \mathrm{GHz}$ (channel 11 of 802.15.4),

(ii) AM/FM simultaneous modulation, 


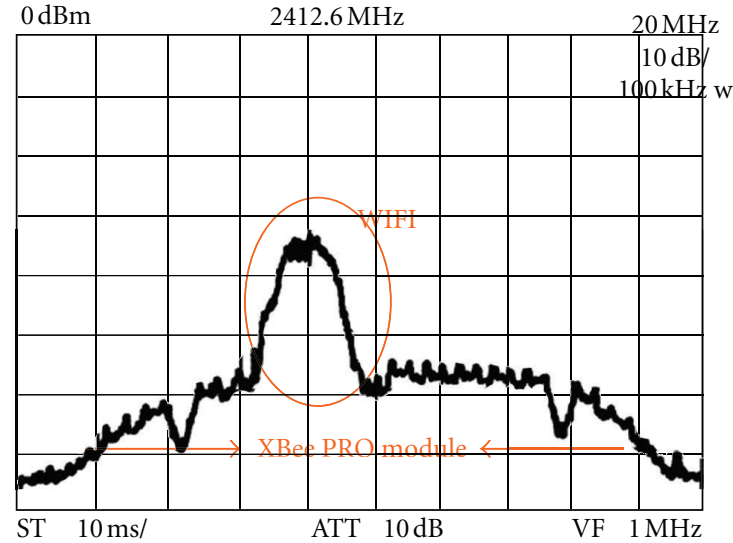

FIgURE 18: Frequency spectrum.

(iii) signal bandwidth: $40 \mathrm{kHz}$,

(iv) AM modulated signal frequency: $20 \mathrm{kHz}$,

(v) power generator from $-20 \mathrm{dBm}$ to $-10 \mathrm{dBm}$.

The second type of noise is WiFi signal irradiated over the WSN with a power level of $+14 \mathrm{dBm}$. The noise was transmitted through channel 1 of the WiFi frequency spectrum. Figure 18 depicts the frequency spectrum used by the XBee PRO module to communicate and the one of WiFi noise. Observing this figure, it is possible to see the superposition of the two spectrums.

In the following, experiments aiming to evaluate the TPSO technique's efficiency in the presence of noise have been performed. It is worth noting that noise was applied only during the second half of the 50 sessions that compose an experiment. In more detail, during the sessions numbered 0 to 24 the noise generator was switched off, and during the sessions numbered 25 to 49 the noise generator was switched on and applied different levels of AM/FM noise as well as WiFi.

\section{Experiments Using AM/FM Noise}

Figure 19 shows the impact of the AM/FM noise of -10d BM on the WSNs Ef. In more detail, each point in the graph represents a specific XBee PRO module transmission power level from 0 to 4 during the 50 sessions (axis $x$ ) against the WSNs average $E f$ (axis $y$ ). For instance, when the XBee PRO modules are transmitting with level $0(10 \mathrm{dBm})$, the whole WSN consumed 6.33 mW.s, which resulted in a WSN efficiency of $36.80 \%$ without EMI. Similarly, the efficiency rises to $93.96 \%$ for the WSN operating in a noise-free environment.

The WSNs average Ef mentioned above has been calculated as arithmetic average of the values of the WSNs Ef during the 50 performed sessions. Regarding the WSNs energy consumption value, it has been obtained throughout the sum of the energy consumed during the 50 sessions. Thus, considering the transmission power level 0 , it is possible to see that the noise drastically affects the WSNs $E f$, since the average has been reduced from about $93.96 \%$ to $36.8 \%$ when

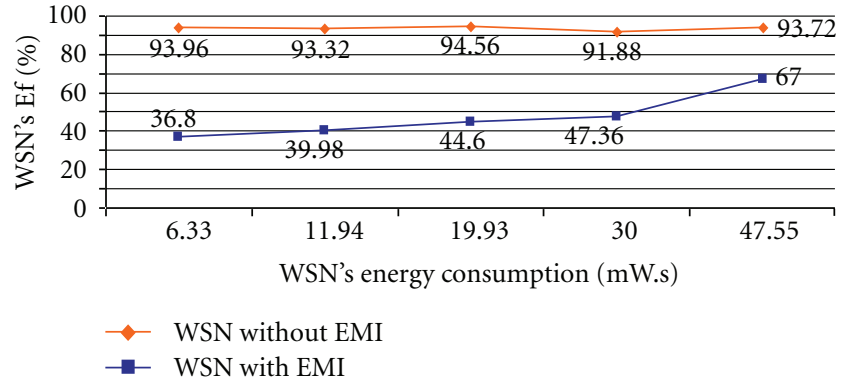

FIGURE 19: Scenario 3. EMI influence on WSNs Ef applying AM/FM noise of $-10 \mathrm{dBm}$.

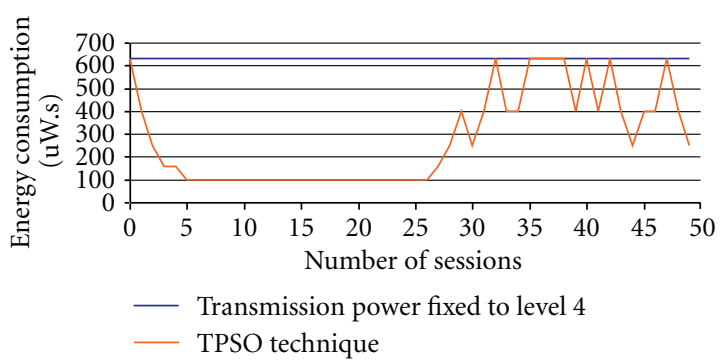

Figure 20: Scenario 3. Comparison of the energy consumption yielded by the TPSO technique with respect to WSN operating with transmission power fixed to level 4 .

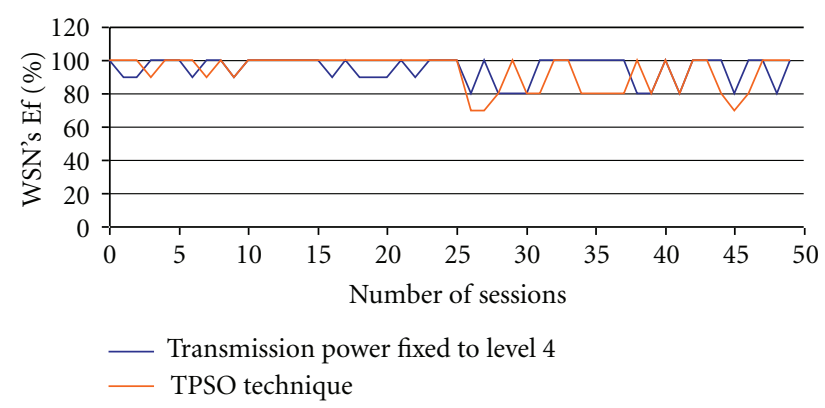

FIgure 21: Scenario 3. Comparison of the WSNs Ef of the TPSO technique with respect to WSN operating with the transmission power fixed to level 4 .

exposed to EMI. Indeed, we can observe that the reduction of the WSNs $E f$ is smaller when the WSN is configured to transmit at the transmission power level $4(18 \mathrm{dBm}$, which represents the maximum transmission power level that the WSN can adopt). In this case, the reduction of the WSNs Ef has been from $93.72 \%$ to $67.00 \%$.

In order to better illustrate the effectiveness of the proposed self-optimization algorithm, Figures 20 and 21 depict the WSNs Ef and the WSNs total energy consumption considering the following characteristics: (1) the WSN has been set to communicate the transmission power level fixed to 4 and (2) the WSN adopting the TPSO algorithm has been configured to target $90 \%$ of WSNs Ef. In this case, the effectiveness of the TPSO technique is clearly visible since the self-optimization algorithm is able to guarantee 


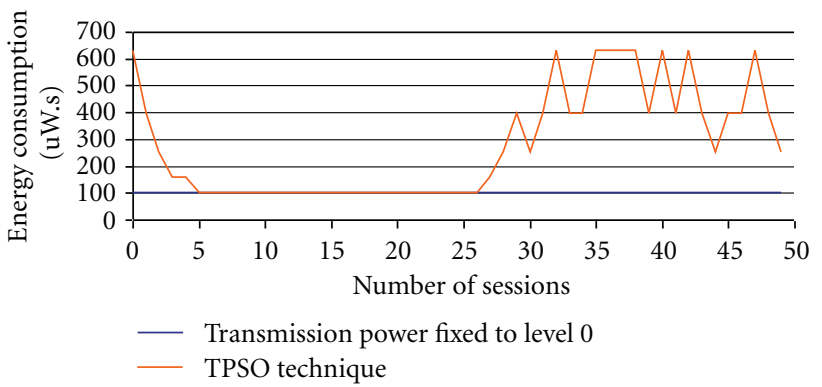

Figure 22: Scenario 3. Comparison of the energy consumption of the TPSO technique with respect to WSN operating with the transmission power fixed to level 0 .

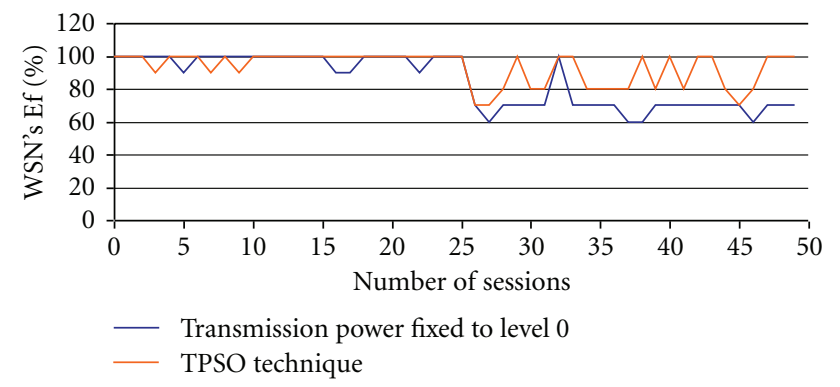

Figure 23: Scenario 3. Comparison of the WSNs Ef of the TPSO technique with respect to WSN operating with the transmission power fixed to level 0 .

the WSN' $E f$ as defined by the target_Ef (90\%) while reducing the energy consumption by $55.6 \%$. In other words, the TPSO algorithm increases the transmission power level in order to compensate for the decrease of the WSNs $E f$ as response to the high AM/FM noise applied on the system (starting from session 25), so that to assure the target_Ef. In this scenario, the WSN' $E f$ for transmission power level 4 is $94.6 \%$ (with an average energy consumption per session equal to $631 \mathrm{uW} . \mathrm{s}$ ), whereas for the TPSO technique, the WSN' $E f$ rendered $93.2 \%$ (with an average consumption of 279.7 uW.s).

Figures 22 and 23 show that the self-optimization algorithm increases transmission power level (starting from session 25) in order to guarantee the WSNs $E f$ at $90 \%$. Thereby, it is demonstrated the effectiveness of the proposed technique. These figures depict the WSNs Ef and energy consumption between the WSN configured to transmit with the minimum transmission power level and the WSN implemented with the proposed approach. Observing such figures, it is possible to conclude that WSNs Ef associated to the network with the TPSO approach achieves an Ef of $93.2 \%$ while the WSN without the self-optimization algorithm reaches an $E f$ of $84 \%$. Indeed, the higher WSNs Ef seen in Figure 23 has been achieved with an increase of about $170 \%$ of energy consumption.

\section{Experiments Using WiFi Noise}

Figures 24-27 demonstrate how the WSNs efficiency changes in the presence of WiFi noise. Figure 24 compares the

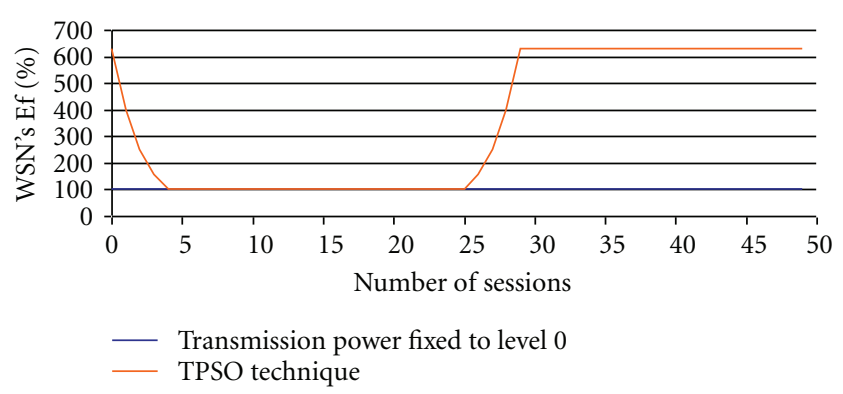

Figure 24: Scenario 3. Comparison of the energy consumption of the TPSO technique with respect to WSN operating with the transmission power fixed to level 0 .

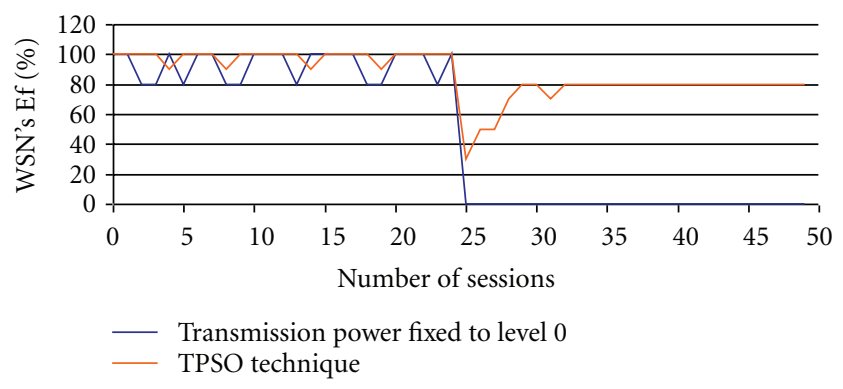

Figure 25: Scenario 3. Comparison between the WSNs Ef obtained using transmission power level fixed to 0 and the TPSO algorithm.

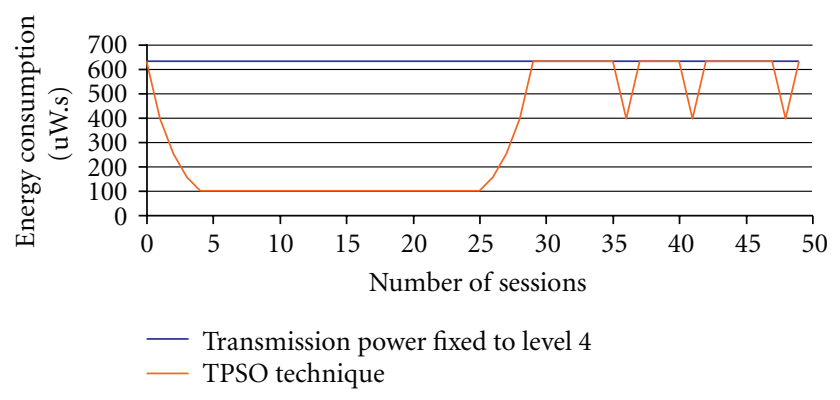

Figure 26: Scenario 3. Comparison of the energy consumption of the TPSO technique with respect to WSN operating with the transmission power fixed to level 0 .

energy consumed by a WSN in two different moments: (1) configured to transmit at the lowest power level available in the XBee PRO modules; and (2) with the WSN making use of the TPSO technique.

Figure 25 depicts the $E f$ of the WSN at two moments, one with the transmission power level fixed to 0 , and one based on the TPSO technique. The WSN with the fixed transmission level reaches an average $E f$ of $46.4 \%$, while the network based on the TPSO technique achieves $86.6 \%$. In order to reach higher communication efficiency for the WSN, the TPSO technique switches to higher transmission power levels to cope with the introduced noise. This yields a $253 \%$ more energy consumed.

In Figures 26 and 27, we observe the results of the energy consumption and the efficiency of a WSN with a 


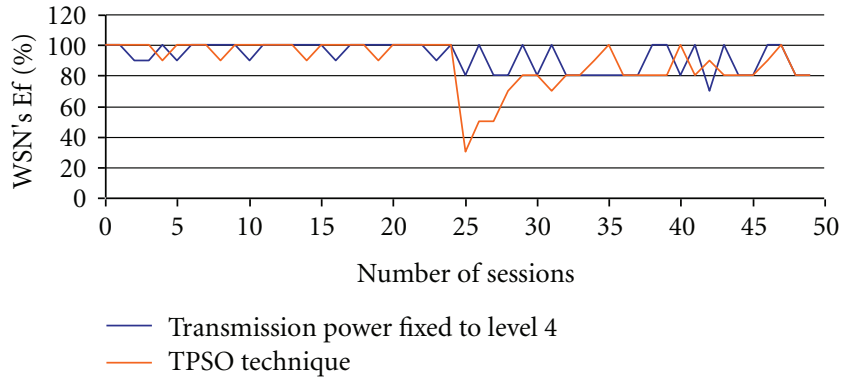

Figure 27: Scenario 3. Comparison of the WSNs Ef of the TPSO technique with respect to WSN operating with the transmission power fixed to level 4 .

transmission power fixed to the maximum level in comparison with a network adopting the TPSO approach. In more detail, Figure 26 shows the power consumed by the WSN to communicate, while Figure 27 shows the WSNs Ef.

Observing Figure 26, we can clearly observe that the proposed approach reduces the transmission power level during the noise-free period (between sessions 0 and 25) and, thereby, dramatically reduces the overall WSN energy consumption. In detail, the technique is able to reduce the energy consumption by $46.12 \%$ along with the execution of the 50 sessions. The obtained results depicted in Figure 27 show that the average $E f$ drops from $92 \%$ to $89 \%$ when comparing the fixed transmission power level with the TPSO technique. Therefore, the proposed approach is able to reduce the power consumption to almost a half while reducing the WSNs $E f$ by only $3 \%$. Indeed, it is important to underline that the WSNs $E f$ is still in between the tolerance interval predefined for these experiments, which is $5 \%$ higher or $5 \%$ lower than the WSNs target Ef.

\section{Final Considerations}

In this paper, we present a transmission power self-optimization technique that aims at guaranteeing a given WSNs $E f$ while trying to reduce the energy consumption required to transmit data between nodes. Thus, the main idea behind the proposed algorithm is to optimize energy consumption when the environment inherent noise is low and to guarantee the connectivity and reliability of the transmitted data when the noise is high. In more detail, the self-optimization algorithm uses the information computed by the MN to adjust the transmission power level associated to each SN. Consequently, the self-optimization algorithm assures the compromise between WSNs Ef and energy consumption. In this scenario, the adoption of the TPSO technique increases the lifetime of WSNs in noisy environments without affecting their QoS.

Experimental results demonstrated the convenience of using the self-optimization algorithm instead of setting a fixed transmission power level available in the XBee PRO modules. When a WSN without the TPSO technique is considered, the transmission power is set at the beginning of the communication and remains the same during its entire network lifetime. This characteristic can be negative considering a WSN in a real environment where the inherent noise is not constant.

Due to the fact that the inherent environment noise is completely variable and random, the TPSO technique will always guarantee the lowest possible transmission power during the communication, while ensuring the target Ef when it is feasible. In this sense, if the noise is too high, the self-optimization algorithm will try to increase the transmission power level until reaching the targeted $E f$ or the transmission power's saturation. Reversely, if the noise is low, the TPSO technique adjusts the transmission power to the lowest possible level that guarantees the WSNs target_Ef. For battery-powered systems, the use of the proposed technique is very rewarding and, sometimes, the unique possible solution to ensure network's lifetime beyond minimum boundaries.

\section{Acknowledgment}

This work has been partially supported by the Brazilian National Science Foundation (CNPq) under contract number 490547/2007-2009.

\section{References}

[1] I. F. Akyildiz, W. Su, Y. Sankarasubramaniam, and E. Cayirci, "A survey on sensor networks," IEEE Communications Magazine, vol. 40, no. 8, pp. 102-105, 2002.

[2] A. Bhrathidasan and V. A. S. Ponduru, "Sensor networks: an overview," IEEE Potentials, vol. 22, no. 2, pp. 20-23, 2003.

[3] A. R. Pinto, M. Camada, M. A. R. Dantas, C. Montez, P. Portugal, and F. Vasques, "Genetic machine learning algorithms in the optimization of communication efficiency in wireless sensor networks," in Proceedings of the 35th Annual Conference of the IEEE Industrial Electronics Society (IECON '09), pp. 2448-2453, November 2009.

[4] S. Panichpapiboon, G. Ferrari, and O. K. Tonguz, "Optimal transmit power in wireless sensor networks," IEEE Transactions on Mobile Computing, vol. 5, no. 10, Article ID 1683791, pp. 1432-1446, 2006.

[5] F. Wang, M. T. Thai, Y. Li, X. Cheng, and D. Z. Du, "Faulttolerant topology control for all-to-one and one-to-all communication in wireless networks," IEEE Transactions on Mobile Computing, vol. 7, no. 3, pp. 322-331, 2008.

[6] C. A. Boano, Z. He, Y. Li, T. Voigt, M. Zuniga, and A. Willig, "Controllable radio interference for experimental and testing purposes in wireless sensor networks," in Proceedings of the 4th International Worshop on Pratical Issues in Building Sensor Network Applications (SenseApp '09), 2009.

[7] F. Lavratti, A. R. Pinto, D. Prestes, L. Bolzani, F. Vargas, and C. Montez, "Towards a transmission power self-optimization in reliable wireless sensor networks," in Proceedings of the 11th Latin-American Test Workshop (LATW'10), March 2010.

[8] F. Lavratti, A. R. Pinto, L. Bolzani et al., "Evaluating a transmission power self-optimization technique for WSN in EMI environments," in Proceedings of the 13th Euromicro Conference on Digital System Design: Architectures, Methods and Tools (DSD '10), pp. 509-515, September 2010.

[9] J. A. Stankovic, T. F. Abdelzaher, C. Lu, L. Sha, and J. C. Hou, "Real-time communication and coordination in embedded 
sensor networks," Proceedings of the IEEE, vol. 91, no. 7, pp. 1002-1022, 2003.

[10] J. O. Kephart and D. M. Chess, "The vision of autonomic computing," Computer, vol. 36, no. 1, pp. 41-50, 2003.

[11] XBee/XBee-PRO OEM RF Modules, http://www.digi.com.

[12] IEEE 802.15.4 Standard Specification, 2009, http://www .ieee802.org/15/pub/TG4.html.

[13] Electromagnetic Compatibility (EMC)—part 4-29: Testing and measurement techniques-Voltage dips, short interruptions and voltage variations on d.c. input power port immunity tests (1st Edition) Normative, 2000-2008, http://www.iec .ch.

[14] F. Vargas and D. P. Prestes, "Conducted electromagnetic noise generator for testing integrated circuits," Patent INPI number PI0705324-0 INPI, 2007, http://www.inpi.gov.br. 

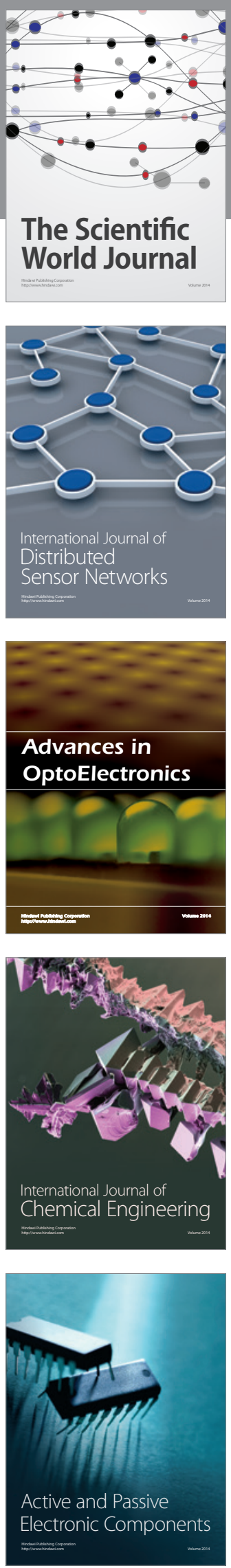
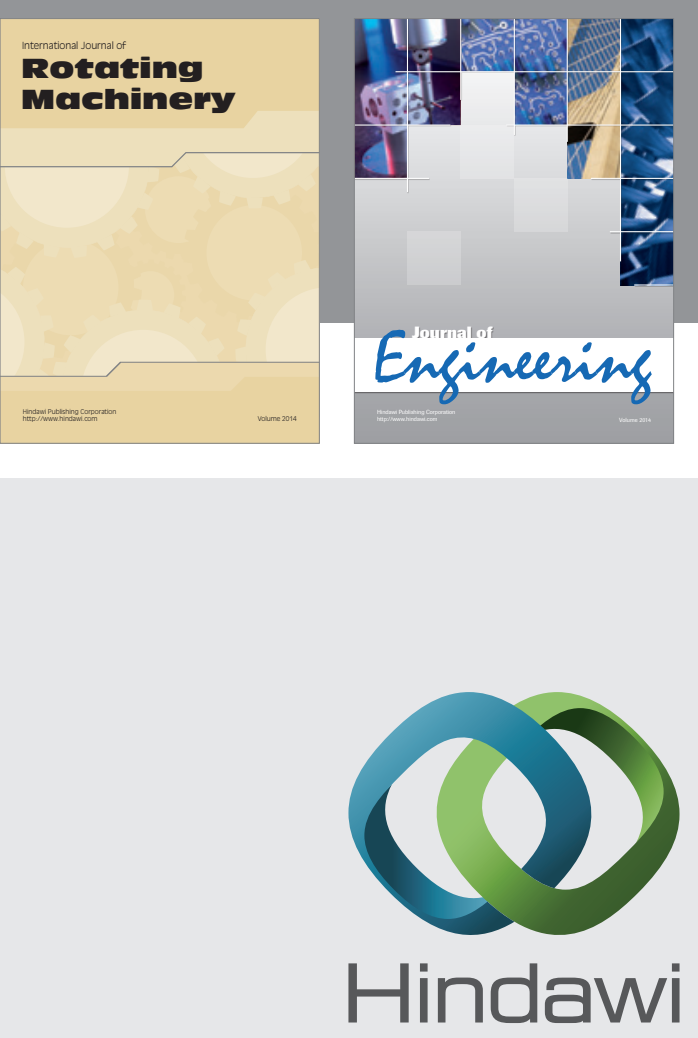

Submit your manuscripts at

http://www.hindawi.com
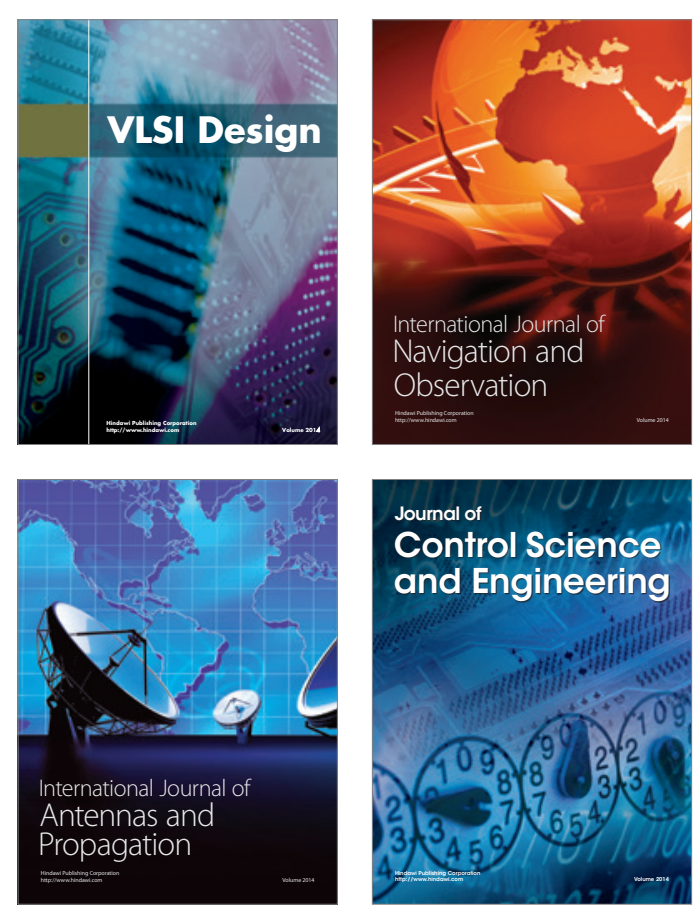
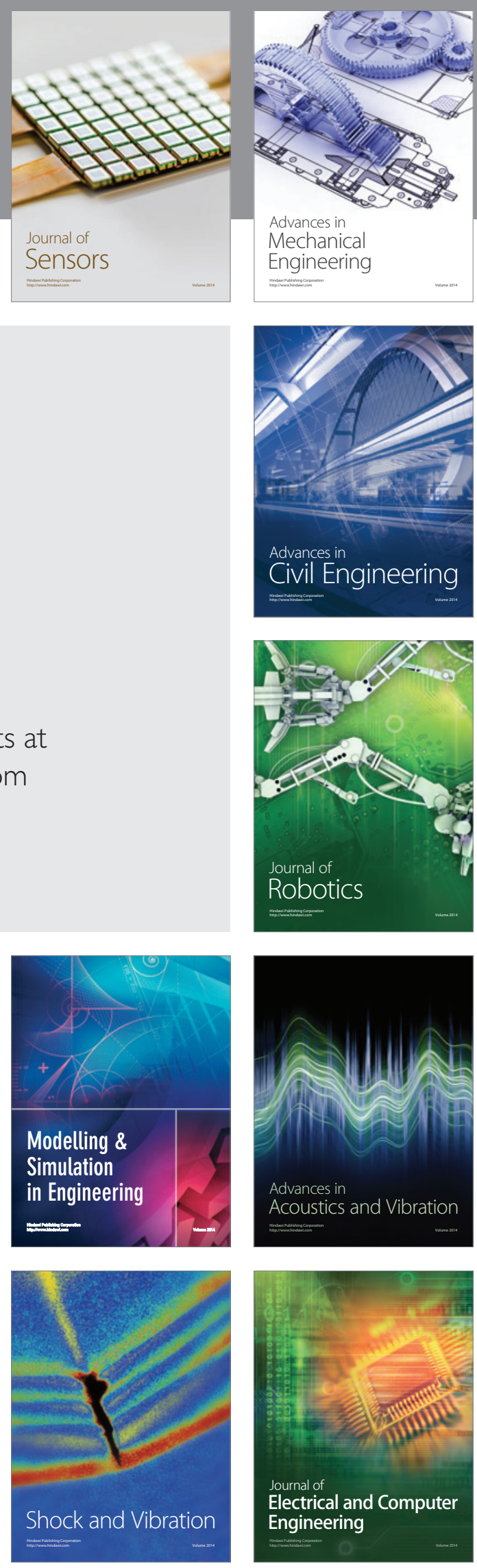